\title{
Flexibility Scheduling for Distribution Systems: A Case Study in Austria
}

\author{
Da Huo ${ }^{1}$, Marcos Santos ${ }^{1}$, Jim McGrath ${ }^{2}$, Neal Wade ${ }^{1}$, David Greenwood ${ }^{*}$ \\ ${ }^{I}$ School of Engineering, Newcastle University, NE1 7RU, UK \\ ${ }^{2}$ Digital Institute, Newcastle University, NE1 7RU, UK \\ *david.greenwood@newcastle.ac.uk
}

\begin{abstract}
Keywords: BATTERY STORAGE, CONVEXIFICATION, DISTRIBUTION SYSTEM, FLEXIBILITY SCHEDULING.
\end{abstract}

\begin{abstract}
Distribution systems could face imminent network constraints violation due to the increasing penetration of uncertain renewable generation and uptake of electric vehicles. This phenomenon could be particularly observed in Austrian distribution systems, because the voltage of each bus must be within $\pm 2 \%$ of the nominal value, according to Austrian network regulations. These constraints can be addressed using flexible resources, such as Battery Energy Storage System, Electric Vehicles (EV), and residential building energy management systems operated to optimally access their latent flexibility. The scheduling of flexible resources yields a non-convex, stochastic, multi-period problem due to the modelling of non-linear power flow equations, uncertainty in forecast errors, and the time-dependent nature of flexible resources. This paper proposes an optimal scheduling scheme, which employs a convexification method to pursue the global minimum with an efficient computation, and applied 2point estimate method to model uncertainty. The method is demonstrated on an Austrian distribution network.
\end{abstract}

\section{Introduction}

\subsection{Background and Literature review}

Electricity distribution systems are under pressure caused by increasing penetration of renewable generation and anticipated growth of customer demand, which can lead to network congestions. Flexible resources, such as Battery Energy Storage Systems (BESS), Electric Vehicles (EV), and residential buildings can be optimally operated to adequately utilise their redundant flexibility, so as to address the above challenges and reduce cost [1]. Specifically, BESS can provide Distribution System Operator (DSO) services such as peak demand shaving and other ancillary services [2].

The DSO can optimally schedule the operations of the flexible assets to minimise costs or meet other objectives. The operations of flexible assets and their scheduling studies in distribution systems have been investigated in the literature as follows: reference [3] proposes the concept of deliverable energy flexibility of distribution system buses, quantifying the available aggregated flexibility offered to the day-ahead energy market; reference [4] presents real-time scheduling schemes for aggregated deferrable loads and storage; reference [5] proposes a scheduling framework at the appliance level for demand peak reduction considering the consumers' flexibility and socio-technical factors; reference [6] optimally schedules the integrated electricity and gas network coupled by energy hubs to maximumly utilise the flexibility in both energy vectors, which is solved by a decomposed particle swarm optimisation approach; the flexibility of Electric Vehicles Aggregators (EVA) is evaluated in reference [7] considering the EVA acting as an energy reserve.
Uncertainty in consumers behaviour or renewable generator outputs can also affect the scheduling of flexible assets, the DSO needs to find a robust scheduling for the system considering the impact of uncertainty, to guarantee meeting the network constraints. In previous literature, reference [8] applies the two-stage stochastic programming to schedule the power flow and flexibility service from reserved distributed energy resources; reference [9] proposes to employ a distributionally robust chance constrained programming to schedule the home appliances considering intermittent renewable generation; reference [10] applies robust optimisation to schedule the micro-grid operations to enhance system resilience considering the uncertainty of market price, load, generation, and islanding duration; reference [11] propose a uncertainty transferring scheme between EVAs in distribution systems, and applies distributionally robust optimisation to mitigate the impact of uncertainty forecast errors. These methods are effective, but computationally intensive ways to include uncertainty in the scheduling process An alternative, the 2-Point Estimate (2PEM) is effective and straightforward to model representative scenarios for uncertainty, it is applied in reference [12] to simulate the uncertainty of renewable generators in residential energy hubs.

\subsection{Contributions and Paper Organisation}

The Austrian Güssing distribution system faces imminent network constraints violation because: (1) the voltage at each bus is within $\pm 2 \%$ of the nominal value according to Austrian network regulations [13]; (2) the increasing penetration of renewable generators and EV uptake causes fluctuation in network power flow and voltage. In order to address the above challenges and following the research scope of Merlon project 
[14], the DSO of Güssing distribution system, Energie Güssing, has installed a BESS and incentivised customer flexibility within the system [15], and proposes to manage these assets to reduce cost while meeting the network constraints. This paper helps address these issues through the following contributions:

- A day-ahead scheduling scheme is proposed to manage the network constraints at minimum cost by optimally controlling the operations of BESS and aggregated flexibility in the system. The uncertainty of load forecast error is modelled by 2PEM.

- The convex relaxed branch flow model presented in [16], in which the exactness of the convexification has been guaranteed, is applied in this paper to model the power flow of the distribution system. It enables an accurate solution with efficient computation.

- The scheduling study is demonstrated on the Austrian Güssing distribution as part of the Merlon project.

The rest of the paper is structured as follows. Section 2 presents the convexification of power flow equations and the modelling of flexible assets. Section 3 formulates the optimal scheduling problem. The case study is investigated in section 4. Section 5 concludes the paper.

\section{Distribution System Modelling}

\subsection{Convexification of Power Flow Model}

The relation between the voltages $V_{i}$ and $V_{j}$ of two adjacent buses $i$ and $j$ can be expressed as follows according to Ohm's law:

$V_{i}-V_{j}=z_{i j} I_{i j}$

where $z_{i j}$ and $I_{i j}$ are the impedance and current of the branch between buses $i$ and $j$. The complex branch power flow $S_{i j}$ is formulated as:

$S_{i j}=V_{i} I_{i j}^{*}$,

where $I_{i j}^{*}$ means the complex conjugate transpose of the current $I_{i j}$. The power balance of a bus $j$ is expressed as:

$\sum_{k: j \rightarrow k} S_{j k}-\sum_{i: i \rightarrow j}\left(S_{i j}-z_{i j}\left|I_{i j}\right|^{2}\right)+y_{j}^{*}\left|V_{j}\right|^{2}=s_{j}$,

where $y_{j}^{*}$ indicates the complex conjugate transpose of shunt admittance from bus $j$ to the ground, $s_{j}$ represents the net complex power injection of bus $j$, quantified by generation minus demand.

According to [16], by defining ancillary variables $\ell_{i j}:=\left|I_{i j}\right|^{2}$ and $v_{i}:=\left|V_{i}\right|^{2}$, and combining (1)-(3), the following equations can be derived:

$$
\begin{aligned}
& p_{j}=\sum_{k: j \rightarrow k} P_{j k}-\sum_{i: i \rightarrow j}\left(P_{i j}-r_{i j} \ell_{i j}\right)+g_{j} v_{j}, \forall j \\
& q_{j}=\sum_{k: j \rightarrow k} Q_{j k}-\sum_{i: i \rightarrow j}\left(Q_{i j}-x_{i j} \ell_{i j}\right)+b_{j} v_{j}, \quad \forall j \\
& v_{j}=v_{i}-2\left(r_{i j} P_{i j}+x_{i j} Q_{i j}\right)+\left(r_{i j}^{2}+x_{i j}^{2}\right) \ell_{i j}, \forall i, j
\end{aligned}
$$

$\ell_{i j}=\frac{P_{i j}^{2}+Q_{i j}^{2}}{v_{i}}, \forall i, j$

where $p_{j}$ and $q_{j}$ are the net active and reactive power injections of bus $j, g_{j}$ and $b_{j}$ are the real and imaginary parts of the shunt admittance between bus $j$ and ground, $P_{i j}$ and $Q_{i j}$ are the active and reactive power flows from bus $i$ to $j, r_{i j}$ and $x_{i j}$ are the resistance and reactance of the branch between bus $i$ and $j$. (7) can be further relaxed to (8), forming the second order cone constraint:

$\ell_{i j} \geq \frac{P_{i j}^{2}+Q_{i j}^{2}}{v_{i}}, \forall i, j$

Equations (4)-(6), (8) represent a convex formulation of the non-convex power flow model, a global minimum can be obtained by using commercially available solvers, the exactness of the relaxation has also been verified in [16].

\subsection{Battery Energy Storage System Model}

The BESS serves as a flexible asset because it can controllably charge and discharge. The available flexibility from the battery storage is dependent on its state of charge, which is determined by its actions in the preceding time step; consequently, the available flexibility is quantified within the scheduling module itself using state of charge data received from the battery. The battery energy balance at each time step, described in terms of the active charging $E_{c h}$ and active discharging energy $E_{d i s}$, is formulated as:

$E_{b}(t)=E_{b}(t-1)-E_{b}^{s t b}+E_{c h}(t) \cdot \eta_{c h}-E_{d i s}(t) / \eta_{d i s}$

where $E_{b}$ indicates the battery energy level, $E_{b}^{s t b}$ is the battery standby loss, $\eta_{c h}$ and $\eta_{d i s}$ denotes the battery charging and discharging efficiency.

The battery storage can also import and export reactive power, which follows the relationship of:

$E_{r p}(t)^{2}+\left(E_{c h}(t)-E_{d i s}(t)\right)^{2} \leq{S_{b}}^{2}$,

where $E_{r p}$ denotes the battery charging reactive power (positive is to import, negative to export), and $S_{b}$ means the apparent power of the battery.

\subsection{Global Flexibility Manager}

In addition to the BESS, other flexible assets identified in the Austrian Güssing distribution system are residential buildings and Electric Vehicles (EV). Residential buildings present flexibility due to the permissible boundary of preserving users' comfort in adjusting the usage of HVAC, domestic hot water, and lights. EV's flexibility is derived by managing EV's operations in coordination with meeting users' desirable state of charge level (SOC) at departure. The Local Flexibility Manager (LFM) collects the flexibility information of each individual domestic building and EV, which are transmitted to, and further aggregated by, the Global Flexibility Manager (GFM). The GFM collects the flexibility offers and submits to the flexibility market, which will be available for the scheduling module. Additionally, the GFM sends the 
aggregated flexibility of each load bus over the scheduling time period to the scheduling module, which contains:

$F L_{\mathrm{GFM}}=\left[L_{i}(t), F L_{\mathrm{up}, i}(t), F L_{\mathrm{dn}, i}(t)\right], \forall i, \forall t$

Where $F L_{\mathrm{GFM}}$ represents the vector of flexibility information sent to the scheduling module by GFM, $L_{i}(t)$ is the baseline load of bus $i$ at time step $t, F L_{\mathrm{up}, i}(t)$ and $F L_{\mathrm{dn}, i}(t)$ are absolutely values of the upward and downward flexibility of bus $i$ at time step $t$.

\section{Scheduling Problem Formulation}

The scheduling problem for the Austrian Güssing distribution system is formulated in this section with the objective of minimising system total cost subject to the constraints of the network and flexible resources. The impact of uncertain load forecast errors is modelled via scenarios with 2PEM based on historical data. The objective function of the scheduling problem is formulated as:

Minimise $\quad C_{\mathrm{TC}}=\sum_{s n=1}^{N} P r_{s n} \cdot \sum_{t=1}^{T}\left\{P_{G, s n}(t) \cdot \Pi_{G}(t)+\right.$ $\sum_{i=1}^{R} P_{r e, i, s n}(t) \cdot \Pi_{r e, i}(t)+\sum_{i=1}^{F}\left[U t_{u p, i, s n}(t)+U t_{d n, i, s n}(t)\right] \cdot$ $\left.\Pi_{f}(t)\right\}$,

where $T$ is the total number of time steps of the scheduling period, $\mathrm{N}$ is the number of scenarios $R$ is the number of renewable generators in the distribution system, $F$ is number of load buses that contain flexible assets; $s n$ and $t$ denote the indices of scenarios and time steps respectively; $P r_{s n}$ is the probability of scenario $s n ; P_{G}$ and $P_{r e, I}$ are the amounts of electricity imported from the grid and the $i^{\text {th }}$ renewable generator; $\Pi_{G}(t)$ and $\Pi_{r e, i}(t)$ indicate the wholsesale and $i^{\text {th }}$ renewable generator's electricity prices at time step $t$, and $\Pi_{f}(t)$ denotes the cost of utilising flexibility at time step $t$. Using the flexibility from the domestic buildings and EVs incurs the cost of flexibility, where the unit price of flexibility should be adopted from the flexibility market $\left(\Pi_{f}(t)\right)$.

The decision variables to be optimised for the scheduling problem contain active and reactive power injections from the grid and each renewable generation for all time steps, ancillary variables $v_{i}$ and $\ell_{i j}$, active and reactive power flows for all time steps, battery charging energy, discharging energy and battery energy level for all time steps, the utilised upward and downward flexibility for all time steps. They are contained in the vector $D e$ in equation (13):

$$
\begin{aligned}
& D e=\left[v_{i, s n}(t), \ell_{i k, s n}(t), P_{i k, s n}(t), Q_{i k, s n}(t), P_{G, s n}(t),\right. \\
& P_{r e, i, s n}(t), E_{c h, s n}(t), E_{d i s, s n}(t), E_{b, s n}(t), U t_{u p, i, s n}(t), \\
& \left.U t_{d n, i, s n}(t)\right] \quad \forall i, \forall k, \forall t, \forall s n
\end{aligned}
$$

Overall, the scheduling problem is formulated by optimising (13) to minimise (12), subject to (4)-(6), (8)-(11), and network constraints including voltage limitation.

\section{Case Study}

\subsection{Summary of Güssing distribution network}

The proposed scheduling scheme is demonstrated using a case study based on the Güssing distribution network in Austria as part of the Merlon project [14]. The schematic of the network is shown in Fig. 1.

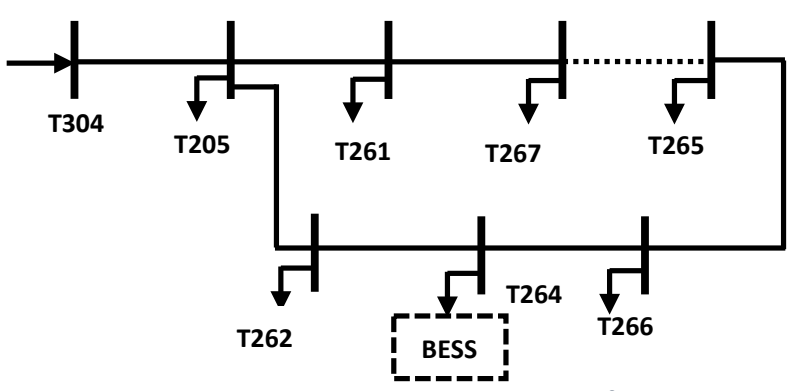

Fig. 1 20-kV Güssing distribution system with the feeder connecting to bus T304.

As seen, the $20-\mathrm{kV}$ distribution network is supplied by a feeder connected with bus T304. The BESS is connected at bus T264, the capacity and the power rating of the battery storage are $0.25 \mathrm{MWh}$ and 0.25 MVA, the charging and discarding efficiency are $95 \%$, the operating limit is between 0 and $100 \%$ of SOC. The GFM collects the available flexibility at each bus and flexibility offers including the price of consuming flexibility. This paper assumes a fixed flexibility price of $€ 30 / \mathrm{MWh} ; 10 \%$ of the base load is assumed to be flexible. The scheduling algorithm is implemented for the Güssing distribution system for 24 hours with a 30 minutes time step.

The BESS is assumed to provide electricity price-based arbitrage from 06:00-00:00, and maintain its SOC between $40 \%$ and $60 \%$ from $00: 00-06: 00$ to provide a primary frequency response service to the transmission system. It is assumed that the initial SOC of the battery is $50 \%$, and the final SOC at the end of scheduled 24 hours should also be $50 \%$ in preparation for the next day's scheduled operation. Austrian network regulations state that the voltage at each bus should be within the $\pm 2 \%$ of the nominal value [13]. The branch parameters were provided by the DSO, Energie Güssing. The demand and outputs of renewable generators over the scheduling time window are provided by a forecasting module; the 2PEM is applied to generate scenarios for modelling demand uncertainty and electricity prices are taken from [17].

\subsection{Scheduling Results}

The proposed scheduling scheme is optimised by Gurobi [18] on the platform of Matlab, which requires an average of 1.05 seconds to optimise one scenario on a laptop with i5 processer and 8 GB memory. The optimal scheduled operations are derived by aggregating the results from all scenarios, with each of them multiplied by its relevant probability.

The battery operations over a 24-hour period are shown in Fig. 2 ; the battery energy level and electricity price are shown in (a), and the reactive power injections are shown in (b).

As seen in Fig. 2 (a), the battery storage maintains its SOC between $40 \%$ and $60 \%$ in 00:00-06:00 to be prepared for primary frequency response request. It fully discharges during the two peaks of electricity price at time steps $17,18,37$, and 
38; it fully charges when the electricity price is lower, which fulfils the function of electricity price-based arbitrage to reduce system cost. The battery storage also injects reactive power to offset the excessive reactive power output from the renewable generators, which satisfies (10), to balance the distribution system as seen in Fig. 2 (b).

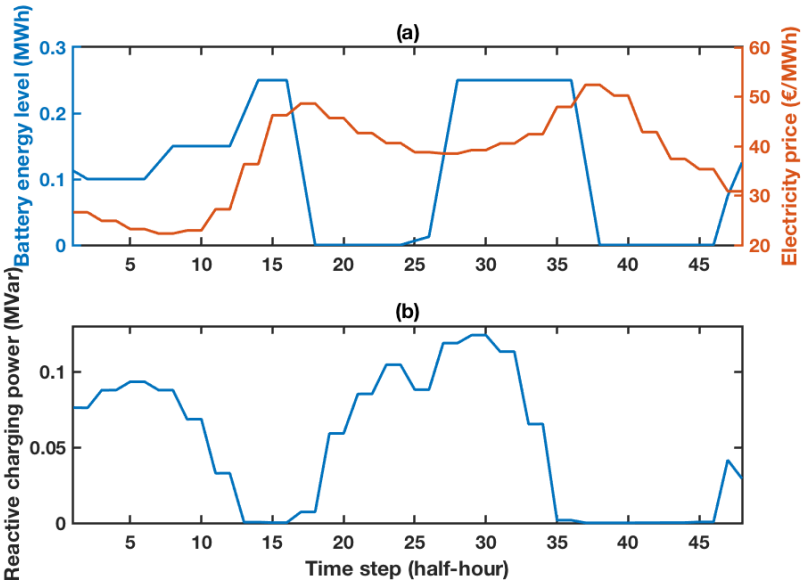

Fig. 2. (a) Electricity price in red and scheduled battery operations in blue; (b) scheduled reactive charging energy

The scheduled utilised flexibility is shown in Fig. 3 with the day-ahead electricity market price and flexibility market price as references.

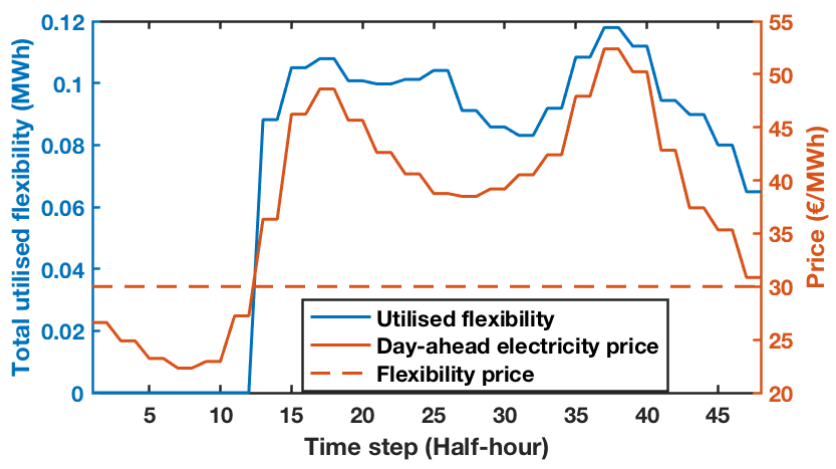

Fig. 3. Scheduled utilised flexibility with day-ahead market and flexibility prices as references

As seen from Fig. 3, the system flexibility is accordingly consumed when the price of using flexibility is lower than importing electricity from the grid or renewable generators, which effectively reduces the system cost.

The voltage of each bus of the distribution network over the scheduling period is shown in Fig. 4.

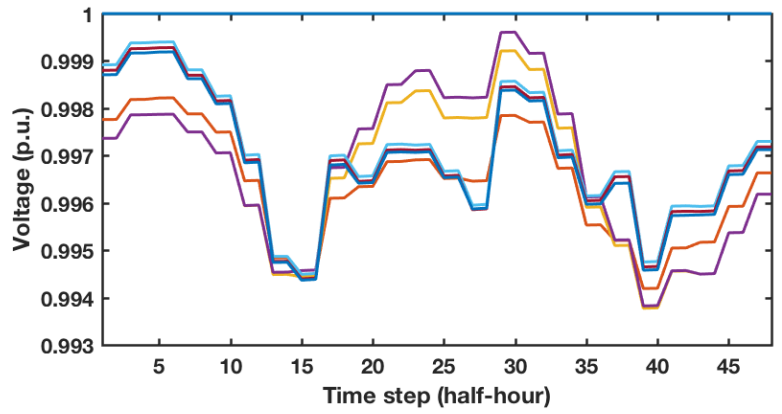

Fig. 4. Voltage of each bus in the Strem area

The voltage with 1 p.u. (per unit, $20 \mathrm{kV}$ is the base voltage) for all time steps represents the slack bus, in this case, bus T304. As seen, the voltages of all buses of the Gussing distribution system are within the boundary of $\pm 2 \%$ compared with the nominal value, hence meets the Austrian transmission system's voltage restrictions.

\subsection{Impacts of Uncertainty and Flexibility}

In order to analyse the impacts of uncertainty and flexible assets to the distribution system scheduling, two comparative scenarios are investigated in this section: scenario 1 uses the proposed scheduling scheme to optimise the network operations but excludes the modelling of uncertainty; scenario 2 applies the 2PEM to simulate the uncertainty without modelling battery storage or other flexible assets in the distribution system, the renewable generators are still modelled. Implementing the proposed scheduling scheme for the system considering both the flexible assets and uncertainty is regarded as scenario 3. The optimal costs of the three scenarios are shown in Table 1.

Table 1 Comparison of costs between scenarios

\begin{tabular}{cc}
\hline \hline Scenario number & Optimal cost $(€)$ \\
\hline 1 & 1516.0 \\
\hline 2 & 1568.9 \\
\hline 3 & 1518.7 \\
\hline \hline
\end{tabular}

By comparing scenarios 2 and 3, it suggests that optimising the operations of flexible assets in the Austrian Güssing distribution system could reduce the total cost by $€ 50.2$ over 24 hours for the analysed case, which also verifies the effectiveness of the proposed scheduling scheme. The cost difference between scenario 1 and 3 is $€ 2.7$, which indicates the extra cost required to hedge the risks or impact of uncertainty during operating the distribution system.

\section{Conclusion}

This paper proposes an optimal scheduling scheme for distribution systems, which optimally manages the network operations including the BESS and other aggregated flexible assets subject to network constraints. The impact of uncertainty is modelled by the 2-point estimate method, a convexification method is applied to relax the complex power flow model to increase the computation efficiency. The 
effectiveness of the scheduling scheme is verified in case study. The main findings are presented as follows:

- Appropriate scheduling of the battery storage and other flexible resources in a distribution system can effectively reduce the energy cost, the reduction is $€ 50.2$ over 24 hours for the analysed Güssing distribution system.

- To avoid violating the network constraints, the impact of uncertainty in distributions system should be properly modelled in the case of high penetration of intermittent customer behaviour and renewable generation.

\section{Acknowledgement}

This work was supported by the European Union's Horizon 2020 research and innovation programme under grant agreement No 824386 .

\section{References}

[1] M. J. Ghadi, A. Rajabi, S. Ghavidel, A. Azizivahed, L. Li, and J. Zhang, "From active distribution systems to decentralized microgrids: A review on regulations and planning approaches based on operational factors," Applied Energy, vol. 253, p. 113543, 2019/11/01/ 2019, doi: https://doi.org/10.1016/j.apenergy.2019.113543.

[2] D. M. Greenwood, N. S. Wade, P. C. Taylor, P. Papadopoulos, and N. Heyward, "A Probabilistic Method Combining Electrical Energy Storage and Real-Time Thermal Ratings to Defer Network Reinforcement," IEEE Transactions on Sustainable Energy, vol. 8, no. 1, pp. 374-384, 2017, doi: 10.1109/TSTE.2016.2600320.

[3] K. Oikonomou, M. Parvania, and R. Khatami, "Deliverable Energy Flexibility Scheduling for Active Distribution Networks," IEEE Transactions on Smart Grid, vol. 11, no. 1, pp. 655-664, 2020, doi: 10.1109/TSG.2019.2927604.

[4] A. Subramanian, M. J. Garcia, D. S. Callaway, K. Poolla, and P. Varaiya, "Real-Time Scheduling of Distributed Resources," IEEE Transactions on Smart Grid, vol. 4, no. 4, pp. 2122-2130, 2013, doi: 10.1109/TSG.2013.2262508.

[5] F. Fanitabasi and E. Pournaras, "Appliance-Level Flexible Scheduling for Socio-Technical Smart Grid Optimization," IEEE Access, vol. 8, pp. 119880-119898, 2020, doi: 10.1109/ACCESS.2020.3001763.

[6] D. Huo, S. Le Blond, C. Gu, W. Wei, and D. Yu, "Optimal operation of interconnected energy hubs by using decomposed hybrid particle swarm and interior-point approach," International Journal of Electrical Power \& Energy Systems, vol. 95, pp. 36-46, 2018/02/01/ 2018, doi: https://doi.org/10.1016/j.ijepes.2017.08.004.

[7] R. Deng et al., "Exploring flexibility of electric vehicle aggregators as energy reserve," Electric Power Systems Research, vol. 184, p. 106305, 2020/07/01/ 2020, doi: https://doi.org/10.1016/j.epsr.2020.106305.

[8] V. A. Evangelopoulos, I. I. Avramidis, and P. S. Georgilakis, "Flexibility Services Management Under Uncertainties for Power Distribution Systems: Stochastic Scheduling and Predictive Real-Time Dispatch," IEEE Access, vol. $8, \quad$ pp. 38855-38871, 2020, doi: 10.1109/ACCESS.2020.2975663.

[9] P. Zhao, H. Wu, C. Gu, and I. Hernando-Gil, "Optimal home energy management under hybrid photovoltaic-storage uncertainty: a distributionally robust chance-constrained approach," IET Renewable Power Generation, vol. 13, no. 11, pp. 1911-1919, 2019, doi: https://doi.org/10.1049/iet-rpg.2018.6169.

[10] N. M. Zografou-Barredo, C. Patsios, I. Sarantakos, P. Davison, S. L. Walker, and P. C. Taylor, "MicroGrid Resilience-Oriented Scheduling: A Robust MISOCP Model," IEEE Transactions on Smart Grid, pp. 1-1, 2020, doi: 10.1109/TSG.2020.3039713.

[11] X. Lu, K. W. Chan, S. Xia, X. Zhang, G. Wang, and F. Li, "A Model to Mitigate Forecast Uncertainties in Distribution Systems Using the Temporal Flexibility of EVAs," IEEE Transactions on Power Systems, vol. 35, no. 3, pp. 2212-2221, 2020, doi: 10.1109/TPWRS.2019.2951108.

[12] M. Rastegar, M. Fotuhi-Firuzabad, H. Zareipour, and M. Moeini-Aghtaieh, "A Probabilistic Energy Management Scheme for Renewable-Based Residential Energy Hubs," IEEE Transactions on Smart Grid, vol. 8, no. 5, pp. 22172227, 2017, doi: 10.1109/TSG.2016.2518920.

[13] "Technische und Organisatorische Regeln für Betreiber und Benutzer von Netzen (TOR), E-CONTROL, available at https://www.e-control.at/recht/marktregeln/tor."

[14] "Integrated Modular Energy Systems and Local Flexibility Trading for Neural Energy Islands (MERLON), available at https://www.merlon-project.eu/."

[15] Da Huo, Marcos V. Santos, David M. Greenwood, Neal S. Wade, Markus P. Resch, "Optimal Battery Sizing for a Distribution Network in Austria to Maximise Profits and Reliability," presented at the CIRED 2020 Berlin Workshop, Berlin, 2020.

[16] M. Farivar and S. H. Low, "Branch Flow Model: Relaxations and Convexification-Part I," IEEE Transactions on Power Systems, vol. 28, no. 3, pp. 2554-2564, 2013, doi: 10.1109/TPWRS.2013.2255317.

[17] "Entsoe Transparency Platform, available at https://transparency.entsoe.eu/dashboard/show.."

[18] "Gurobi Optimization LLC. "Gurobi Optimizer Reference Manual," http://www.gurobi.com." 